\section{Fatal meningitis complicating cryosurgery for haemorrhoids}

Cryosurgery is a common, effective, and safe treatment for haemorrhoids. ${ }^{1-4}$ We describe what we believe to be the first reported case of meningitis complicating cryosurgery for haemorrhoids.

\section{Case report}

A 62 year old woman was admitted for treatment of third degree haemorrhoids that had prolapsed and become painful. She had been troubled by bleeding haemorrhoids for some months. She had received lorazepam and flupenthixol for anxiety and depression for many years. Preoperative assessment showed no abnormality except pallor and prolapsed third degree haemorrhoids. Her preoperative haemoglobin concentration was $9 \mathrm{~g} / \mathrm{dl}$ with iron deficient changes (mean cell volume $76 \mathrm{fl}$; mean corpuscular haemoglobin $24.5 \mathrm{pg}$; mean corpuscular haemoglobin concentration $32.4 \mathrm{~g} / \mathrm{dl}$ ). This anaemia was ascribed to haemorrhoidal bleeding. Because of her pain her haemorrhoids were treated by cryosurgery before her anaemia was investigated.

After sedation with intravenous diazepam a Keymed FT 300 cryoprobe (nitrous oxide) was applied to each haemorrhoidal mass for two minutes. The procedure was uneventful. Next day she was well but uncomfortable. On the second morning after the operation she was found unresponsive in her room.

On examination she was unconscious and responsive only to deep pain with a fever of $39^{\circ} \mathrm{C}$ rectally and pallor. Pulse was 140 beats/minute and regular and blood pressure $140 / 80 \mathrm{~mm} \mathrm{Hg}$. Dullness to percussion and coarse crackles were detected at the base of her right lung. Findings on abdominal examination were normal. Funduses were normal. She had mild neck stiffness, but Kernig's sign was negative. Both pupils were fixed in the mid-position and showed no response to light. Corneal and gag reflexes were absent. Muscle tone and peripheral reflexes were normal and plantar responses downgoing.

Laboratory findings included haemoglobin concentration $8.4 \mathrm{~g} / \mathrm{dl}$ with iron deficient changes and white cell count $16 \cdot 7 \times 10^{9} / 1$ with $94 \%$ neutrophils and $6 \%$ lymphocytes. Chest $x$ ray examination showed patchy consolidation at the base of the right lung. Blood glucose concentration was $4 \cdot 2 \mathrm{mmol} / \mathrm{l}$ $(76 \mathrm{mg} / 100 \mathrm{ml})$, urea $8.4 \mathrm{mmol} / \mathrm{l}(50 \mathrm{mg} / 100 \mathrm{ml})$, and creatinine $69 \mu \mathrm{mol} / \mathrm{l}$ $(780 \mu \mathrm{g} / 100 \mathrm{ml})$. Liver function tests yielded normal results except albumin concentration $22 \mathrm{~g} / \mathrm{l}$. Lorazepam concentration was $2 \mu \mathrm{g} / \mathrm{l}$, and drug screening for amphetamines, barbiturates, codeine, methadone, cyclizine, morphine, and dihydrocodeine yielded negative results. Lumbar puncture produced thin green pus with a protein concentration of $6.4 \mathrm{~g} / \mathrm{l}$. Microscopical examination showed vast numbers of pus cells and polymorphonuclear leucocytes, which, even on dilution, obscured the microscopic field. Thus no organisms could be seen on either Gram or Ziehl-Neelsen staining. Culture showed heavy growths of Escherichia coli and Streptococcus faecalis (both sensitive to gentamicin), Bacteroides fragilis (sensitive to metronidazole), and a microaerophilic streptococcus (sensitive to chloramphenicol). Blood culture grew $B$ fragilis and $S$ faecalis. A laryngeal swab grew Staphylococcus aureus (sensitive to flucloxacillin). A midstream urine specimen showed 25 white cells but no red cells or casts on microscopy and no bacterial growth on culture.

Her coma was ascribed to purulent bacterial meningitis with septicaemia and secondary right basal aspiration pneumonia. She received intravenous benzylpenicillin, flucloxacillin, chloramphenicol, gentamicin (serum concentrations monitored), metronidazole, hydrocortisone, blood transfusion, and physiotherapy but never regained consciousness and died eight days after admission. Postmortem examination confirmed the clinical diagnoses. There was no evidence of gastrointestinal disease, other than the sloughing haemorrhoids, or of solid tissue or haemopoietic malignancy or lymphoma.

\section{Comment}

We postulate that damage to tissue resulting from the cryosurgery provided the portal of entry for the four different colonic organisms responsible for our patient's septicaemia and meningitis. Polymicrobial anaerobic bacterial meningitis due to a meningorectal fistula has been described in a patient with diabetes who had previously received radiotherapy for a rectal adenocarcinoma ${ }^{5}$ but not to our knowledge in a patient with benign anal disease.

In our patient there was no clinical or postmortem evidence of any other portal of entry in the gut, and she was not immunocompromised before undergoing cryosurgery. We think that the right lower lobe pneumonia was secondary to aspiration of gastric contents due to an impaired level of consciousness and that the colonic bacteria causing meningitis were exceedingly unlikely to have gained access to the circulation initially through the lung.

We submit that meningitis must now be accepted as a rare complication of cryosurgery to haemorrhoids.

We thank Mr A E Carter, Dr H B McMichael, and Dr I A Lampert for permission to report on this patient and Mrs J Lucas and Mrs J Poller for secretarial help.

${ }^{1}$ O'Callaghan JD, Matheson TS, Hall R. In-patient treatment of prolapsing piles: cryosurgery versus Milligan-Morgan haemorrhoidectomy. Br 7 Surg 1982;3:157-9.

2 Berry AR, Di Costa EFD. The treatment of haemorrhoids by surgery. f R Coll Surg Edinb 1978;23:37-9.

${ }^{3}$ Smith LE, Goodreau JJ, Fouty WJ. Operative hemorrhoidectomy versus cryodestruction. Dis Colon Rectum 1979;22:10-6.

4 Goligher JC. Cryosurgery for hemorrhoids. Dis Colon Rectum 1976;19: 213-8.

${ }^{5}$ Walsh TJ, Weinstein RA, Malinoff $\mathrm{H}$, et al. Meningorectal fistula as a cause of polymicrobial anaerobic meningitis. Am $\mathcal{F}$ Clin Pathol 1982; 78:127-30.

(Accepted 15 December 1983)

Ealing General Hospital, Middlesex UB1 3HW

JOHN ANDERSON, MA, MRCP, medical registrar

ADRIAN STEGER, FRCSGLAS, surgical registrar

Correspondence to: Dr J V Anderson.

\section{Rumination in bulimia nervosa}

Little is known about habitual rumination and there have been few systematic studies of the phenomenon. An exception was a recent paper describing a series of nine cases. ${ }^{1}$ That paper concluded that since there appeared to be no coexisting physical or psychiatric disorder habitual rumination must be a distinct and benign clinical syndrome. We describe seven patients with this pattern of behaviour, each of whom fulfilled strict diagnostic criteria for bulimia nervosa.

\section{Patients, methods, and results}

Bulimia nervosa is an eating disorder in which recurrent bouts of overeating are accompanied by compensatory dieting, self induced vomiting, or laxative abuse. ${ }^{2} 3$ Various other behavioural and attitudinal abnormalities may be present, including habitual rumination. ${ }^{3}$

We have recently completed a systematic study of this disorder. ${ }^{4}$ Thirty five women (mean age 23.5 (SD 4.4) years) were investigated, each of whom fulfilled conservative operational diagnostic criteria based on those of Russell.2 The patients comprised a consecutive series of referrals from general practitioners in Oxford. Seven of the patients reported habitual rumination: four ruminated every day, and all seven had been ruminating for at least 12 months. Rumination usually began shortly after eating and consisted of the effortless regurgitation of small quantities of food, which would then be chewed. Subsequently the food would be reswallowed or spat out. Rumination tended to occur in private and generally continued for several hours. Some patients regarded it as innocuous, whereas others viewed it with shame and self disgust. Most described the process as soothing, and four reported that the taste of food was improved by a period of "marination" in the stomach. In no case was rumination a presenting complaint; instead, patients complained of having lost control over eating. The practice seemed to have had few serious adverse effects: two patients reported halitosis, two had recurrent dental problems, one described excessive salivation, and one had painless parotid enlargement. Rumination did appear to be a difficult habit to break, however, since four patients described unsuccessful attempts to stop.

Comparison of those who ruminated with the remaining patients disclosed few differences. Both groups had grossly disturbed eating habits, abnormal attitudes to body weight and shape, and high levels of psychiatric morbidity. There were, however, two notable differences: a history of anorexia nervosa was more common among patients who ruminated (four of the seven patients fulfilled Russell's ${ }^{2}$ criteria compared with $18 \%$ of the remainder), and more of the ruminating group had previously received psychiatric treatment for an eating problem (five of the seven patients compared with $21 \%$ of the remainder). 
Six of the seven ruminating patients subsequently received treatment for bulimia nervosa. Those who successfully regained control over eating also stopped ruminating. No specific treatment techniques appeared to be necessary.

\section{Comment}

While habitual rumination may occur in the absence of psychiatric disorder,' our findings indicate that it is present in a substantial minority of patients with bulimia nervosa and that such patients are particularly likely to have had anorexia nervosa in the past. Since the prevalence of bulimia nervosa among young adult women may be in the region of $1^{0}{ }_{0}^{5}$ habitual rumination is probably more common than has been generally thought. Yet even when it occurs as a feature of bulimia nervosa it may remain undetected, since it is not always reported by the patient.

Interestingly, in patients with bulimia nervosa rumination is distinctive because there is a tendency to spit out the regurgitated food at regular intervals. This may be attributed to these patients' desire to avoid absorbing what they have eaten, which in turn is secondary to their profound fear of becoming fat.

We are greatly indebted to Marianne O'Connor for help with the assessment interviews and data analysis. This research was supported by a grant from the Medical Research Council.

' Levine DF, Wingate DL, Pfeffer JM, Butcher P. Habitual rumination: a benign disorder. Br Med $\mathcal{F} 1983 ; \mathbf{2 8 7}: 255-6$.

2 Russell GFM. Bulimia nervosa: an ominous variant of anorexia nervosa. Psychol Med 1979;9:429-48.

${ }^{3}$ Fairburn CG. Bulimia nervosa. Br f Hosp Med 1983;29:537-42.

${ }^{4}$ Fairburn CG, Cooper PJ. The clinical features of bulimia nervosa. $\mathrm{Br} \mathcal{F}$ Psychiatry (in press).

${ }^{5}$ Cooper PJ, Fairburn CG. Binge-eating and self-induced vomiting in the community: a preliminary study. Br 7 Psychiatry $1983 ; 142: 139-44$

(Accepted 15 December 1983)

Department of Psychiatry, University of Oxford, Warneford Hospital, Oxford OX3 7JX

CHRISTOPHER G FAIRBURN, MPHIL, MRCPSYCH, research psychiatrist PETER J COOPER, DPHIL, DIPPSYCH, research fellow in clinical psychology

\section{Initial screening of children with urinary tract infections: is plain film radiography and ultrasonography enough?}

When a child presents with a possible urinary infection general procedures are: taking the clinical history, examination (including examination of urine by microscopy and culture), and treatment with the appropriate antibiotic. A condition requiring an operation may be obvious on examination-for example, urethral valves in a boy with a palpable bladder. If the diagnosis is not clear, however, there is little consensus about the investigations needed in children with proved urinary infections. Some workers argue that such infections are so common that a first episode need not be investigated: any underlying abnormality of the urinary tract will declare itself with a second or third infection. Others press for investigation of all first infections so that nephrons are saved and the diagnosis and prognosis understood. The tests used may be unpleasant (for example, micturating cystography) as well as potentially dangerous (for example, intravenous urography). We carried out a study to test the value of abdominal plain film and ultrasound imaging as screening tests.

\section{Patients, methods, and results}

We prospectively investigated 50 children aged over 6 months who attended hospital with suspected or already diagnosed urinary infections (or other relevant symptoms). All were referred for intravenous urography, most from a paediatric urology clinic (RHW), but others from general paediatric clinics. The findings on the plain film of the intravenous urogram series and on urinary tract ultrasound scanning were judged by one of us
(TS) without knowledge of subsequent tests. Real time ultrasound examination was carried out by two experienced, qualified ultrasonographers, who recorded multiple images. In doubtful cases one of us (TS) took part in scanning. Particular attention was paid to the full bladder and the lower ureters behind it.

The table compares findings on ultrasonography and intravenous urography. The predictive value of normal findings on ultrasound scanning was high $(90 \%)$, but we missed four children with upper urinary tract abnormalities (two with renal scars and no reflux, one with intermittent ureteric distension and possible neuropathy, and one with bilateral reflux whose sibling had reflux). Abnormal findings on the ultrasound scan were less predictive, with an incidence of false positive results of $30 \%$. Such errors are of less concern as they lead only to unnecessary intravenous urography.

Comparison of findings on intravenous urography and ultrasound scanning in 50 children (30 girls, 20 boys, mean age 7 years)

\begin{tabular}{lcc}
\hline \multirow{2}{*}{ Results of ultrasound scanning } & \multicolumn{2}{c}{ Results of intravenous urography } \\
\cline { 2 - 3 } & Abnormal & Normal \\
\hline Abnormal & 7 & 3 \\
Normal & 4 & 36
\end{tabular}

$\ell^{2}$ with Yates's correction $=13.47 ; \mathrm{p}<0.001$

Predictive value of normal finding on ultrasound scanning $=90 \%(36 / 40)$; rate of false negative results $=10^{\circ},(4 / 40)$; specificity $=92^{\circ} \circ(36 / 39)$.

Predictive value of abnormal finding on ultrasound scanning $=70 \%(7 / 10)$; rate of false positive results $=30 \%(3 / 10)$; sensitivity $=64 \%(7 / 11)$

\section{Comment}

Children with urinary tract infections are screened to find surgically remediable causes - that is, urinary stones and congenital anomalies of drainage such as hydronephrosis, megaureter, or duplex lesions. These findings are rewarding compared with the finding of reflux nephropathy, the complex disorder linking vesicoureteric reflux and renal scarring, formerly called chronic pyelonephritis. Two decades ago there was a strong drive for diagnosis of this condition and for reimplanting most refluxing ureters to guard against further or potential renal damage. Subsequently it was established that any renal scarring was already determined when the child first presented. Reflux itself tends to improve spontaneously. ${ }^{2}$ In schoolgirls neither reflux nor covert bacteriuria contribute to the progression of kidney damage. ${ }^{3}$ With conservative measures reflux nephropathy generally runs a benign course, and the role of operation needs critical appraisal $^{4}$ : a recent report suggested that it offers little if any benefit. ${ }^{5}$

Plain film radiography and ultrasound scanning are useful in screening for correctable causes of children's first urinary infections such as renal stones or major drainage anomalies. Some cases of reflux nephropathy will be missed, but such errors may not be clinically important. We do not hesitate to go on to further investigations, including nuclear medicine studies, if there is uncertainty about a diagnosis. We consider that intravenous urography remains a diagnostic touchstone in paediatric urology and use it readily when appropriate. In many children with a first urinary infection, however, this unpleasant test can now be avoided.

We thank Mrs P Farman and Mrs J Lawrence for performing the ultrasonography.

${ }^{1}$ Hodson J. Reflux nephropathy: a personal historical review. American Fournal of Radiology 1981;137:451-62.

${ }^{2}$ Edwards D, Normand ICS, Prescod N, Smellie JM. Disappearance of vesicoureteric reflux during long-term prophylaxis of urinary tract infection in children. $\mathrm{Br}$ Med $\mathcal{f} 1977 ; \mathrm{ii}: 285-8$.

3 Jones KV, Asscher AW, Jones ERV, Mattholie K, Leach $\mathrm{K}$, Thomson GM. Glomerular filtration rate in schoolgirls with covert bacteriuria. Br Med f 1982;285:1307-10.

${ }^{4}$ Kincaid-Smith P. Reflux nephropathy. Br Med F 1983;286:2002-3.

${ }^{5}$ Birmingham Reflux Study Group. Prospective trial of operative versus non-operative treatment of severe vesicoureteric reflux: two years' observation in 96 children. $\mathrm{Br} M e d \mathcal{F} 1983 ; 287: 171-4$.

(Accepted 1 December 1983)

University of Cambridge and Addenbrooke's Hospital, Cambridge CB2 2QQ

THOMAS SHERWOOD, FRCR, FRCP, professor, department of radiology ROBERT H WHITAKER, MCHIR, FRCS, consultant, department of urology

Correspondence to: Professor T Sherwood. 